\title{
Symposium review: Dairy Brain-Informing decisions on dairy farms using data analytics*
}

\author{
Michael C. Ferris, $†$ ๑ Adam Christensen, and Steven R. Wangen \\ Computer Sciences Department and Wisconsin Institute for Discovery, University of Wisconsin-Madison, Madison 53706
}

\section{ABSTRACT}

Management decisions can be informed by near-realtime data streams to improve the economics of the farm and to positively benefit the overall health of a dairy herd or the larger environment. Decision support tools can use data management services and analytics to exploit data streams from farm and other economic, health, and agricultural sources. We will describe a decision support tool that couples data analytics tools to underlying cow, herd, and economic data with an application programming interface. This interface allows the user to interact with a collection of dairy applications without fully exposing the intricacies of the underlying system model and understand the effects of different decisions on outputs of interest. The collection of these applications will form the basis of the Dairy Brain decision support system, which will provide management suggestions to farmers at a single animal or farm level. Dairy operations data will be gathered, cleaned, organized, and disseminated through an agricultural data hub, exploiting newly developed ontologies for integration of multiple data sources. Models of feed efficiency, culling, or other dairy operations (such as large capital expenditures, outsourcing opportunities, and interactions with regulators) form the basis of analytical approaches, operationalized via tools that help secure information and control uncertainties. The applications will be independently generated to provide flexibility, and use tools and modeling approaches from the data science, simulation, machine learning, and optimization disciplines to provide specific recommendations to decision makers. The Dairy Brain is a decision support system that couples data analytics tools with a suite of applications that integrate cow, herd, and economic data to inform management, operational, and

Received July 1, 2019.

Accepted December 23, 2019.

*Presented as part of the Production, Management, and the Environment Symposium: Advancing Artificial Intelligence on Dairy Farms at the ADSA Annual Meeting, Cincinnati, Ohio, June 2019.

†Corresponding author: ferris@cs.wisc.edu animal health improving practices. Research challenges that remain include dealing with increased variability as predictions go from herd or pen level down to individual cow level and choosing the appropriate tool or technique to deal with a specific problem.

Key words: data management and decision support tools, Dairy Brain, machine learning and optimization, application programming interface

\section{INTRODUCTION AND MOTIVATION}

Dairy farms are increasingly embracing technological innovations, which often generate vast amounts of data. The resulting data ecosystem is complex. Integrating and interpreting these data to improve whole-farmbased management and decision support has proven challenging. To be broadly useful, any attempt to develop an interconnected system of data services must incorporate a priori considerations and standards. We describe a system that integrates data from farms and facilitates a suite of dairy applications that interface with that data and provide enhancements using data analytical tools and visualizations in dairy-specific and domain-recognizable formats.

To be successful, data must first be acquired and cleaned in a reliable fashion and provided to the data analytics application in a way that has named the contents in a consistent manner. The following 3 paragraphs demonstrate the next step, 1 of 3 basic categories of data analytics: descriptive, predictive, and prescriptive. Descriptive analytics (such as financial and medical apps) use collected information to tell us what has happened in the past, akin to traditional reporting or summarization. Predictive analytics (such as weather and air quality apps) generally increase the level of analytical sophistication, typically through the application of statistical models that use the same data to forecast what is likely to happen in the future. Prescriptive analytics (such as traffic routing or hydrocarbon locating algorithms) increase the sophistication of analysis further and focus on evaluating which decisions lead to desired outcomes, often using an optimization or simulation computation. 
As a first descriptive example, in banking applications (such as Quickbooks or Wave), financial information is collected and automatically categorized, and summary information, such as cash flow, is presented using standard and user-customized visualizations. Actions such as transfers between accounts, invoicing, accepting credit card payments, and payroll are available but require standards for data taxonomy to implement. Medical information is similar-privacy and security must be maintained but the types of actions needed in this domain are different. Some hospital-scale reporting needs anonymized data, but scheduling patients requires sharing of individual patient data to be effective.

Weather apps act differently, in that they involve large-scale data sources that update in real-time; data and tools are frequently open source or open access. The observed data are used as initializations for a numerical weather prediction model that provides users with ensemble forecasts, an example of predictive analytics. These can work at different timescales and spatial resolutions for different applications, including short-term weather forecasts, transportation guidance, or climate and ocean surface modeling. Air quality models often couple fine-scale weather models with atmospheric transport models.

The third set of examples are prescriptive. Electricity and gas companies rely on weather forecasts to anticipate demand, which can be strongly influenced by temperature, and these are used for optimized dispatch. Integrating various data streams also enables smart homes to receive information about electricity prices and weather forecasts to cool or heat a house at cost-effective times. Apps for ride-hailing services (e.g., Uber or Lyft) use traffic information to determine travel times and road congestion based on real-time, large-scale data acquisition. Additionally, they take private information from riders about trips and use a matching algorithm to pair riders with drivers. This is coupled with a financial transfer carried out in a secure way as outlined above for financial apps.

Other uses occur in medical applications, such as automated treatment recommender systems for doctors to help in patient diagnosis or systems that perform some pattern matching of related symptoms to suggest different treatments or outcomes.

We provide a framework to facilitate decision support in a broad range of dairy farm application areas and show how the above analytics categories fit into our overall model. When designing a system, it is critical that the designer be cognizant of who the system is for, what it aims to do, and how the system should operate. The types of decisions being considered could be strategic in nature (capital expansions, pricing, culling) or operational (logistic, pen management, health predictions). Depending on the nature of the problem, a decision may be required in a yearly, seasonal, daily, or even hourly timeframe. Furthermore, although decision support systems are often viewed as replacements for human decision makers, we prefer to focus on developing data tools that help inform the human-in-the-loop of good strategy and operations.

First, genomic data aside, the dairy farm information that we are collecting is not "big data" in the acknowledged scientific sense. However, the tools and principles we are using are grounded in the methodologies and ideas present in that literature (Wolfert et al., 2017). Second, we have designed this system to be flexible in application and separate the collection, storage, and management of the data (the agricultural data hub, AgDH) from the development and deployment of analytical tools for insights, termed the "Dairy Brain." The Dairy Brain will leverage the aggregated raw data from the farm made available via the $\mathrm{AgDH}$ to provide both predictive analyses that will project farm performance into the future and prescriptive analytics that will inform improved management actions. Avoiding a tightly coupled dependency between the 2 entities helps ensure the long-term utility of our efforts.

Systems that collate information from large numbers of farms could be used for policy or regulation analyses or benchmarking (leading to opportunities for learning from others). In this context, data privacy becomes an issue, and individual farms need to be anonymized effectively so that competitive advantages are not lost (who can see what and when?). Agricultural data initiatives in both the European Union and the United States have proposed data codes of conduct ("best practices"). Documents published by COPA-COGECA (Comité des organisations professionnelles agricolesComité général de la coopération agricole de l'Union européenne; https://copa-cogeca.eu/img/user/files/ EU\%20CODE/EU_Code_2018_web_version.pdf) as well as the American Farm Bureau Federation (https: //www.fb.org/issues/technology/data-privacy/privacy -and-security-principles-for-farm-data) put the farmer at the center of the data ownership question (i.e., farmers own their data and are responsible for managing various contractual arrangements with third parties that might use or reuse their data). Our system is aware of these issues but is currently instrumented to provide advice (in a uniform fashion) to an individual farm, rather than supply any third parties with aggregate or anonymized analyses that could be linked back to specific farms. In doing so, we keep data access limited to the owner and are able to delay a more complete treatment of the privacy issues to a later date when we will elicit input from a network of peers (discussed in Conclusions). The model is intended to act on ongo- 
ing data updates (that are provided at the time scales outlined above), with the decision support outputs also being updated at these time scales.

Dairy farm data tends to be "dirty." In the United States, many farms remain small and have somewhat limited data collection facilities; data cleaning is not a high priority for many farmers due to the lack of a clearly defined value proposition. Automatic data cleaning facilities can enhance the utility of the data, but the transformation process can lead to different entities claiming ownership of the resultant data. These transformation processes may benefit from adopting FAIR (findable, accessible, interoperable, reusable) data principles, resulting in additional data clarity (albeit at the expense of data object complexity; Wilkinson et al., 2016). A coordination innovation network is a mechanism that we suggest should be employed to delineate the issues and suggest mechanisms to overcome ownership and ontology conflicts and privacy concerns, and to engage with research that understands and provides a value proposition.

\section{AGRICULTURAL DATA HUB}

A critical step in the development of this service is the creation of a prototype $\mathrm{AgDH}$ to gather and disseminate multiple data streams relevant to dairy operations. The distributed nature of the data generators (e.g., across farms or platforms) suggests that such an aggregator entity plays a crucial role in providing harmonized data to analytical tools. The dairy market is demanding an $\mathrm{AgDH}$-like entity, as evidenced by resources such as www.mmmooogle.com, www.dairydatawarehouse.com, www.vyla.com, and www.365farmnet.com. However, the details of these offerings suggest that hard work is needed to capture the myriad data (e.g., genetic, nutritional, reproductive, replacement), transform and join these data streams, and provide functional access to the resulting data for use in decision making. As such, we are prototyping an $\mathrm{AgDH}$ service that will collect, harmonize, store, and distribute farm data. The AgDH service will provide permissioned programmatic access to on-farm-collected dairy-specific data.

In our setting, Figure 1 demonstrates the richness and diversity of the data we are capturing, which includes information about milk production, milk component analysis, milk spectral analysis, diet, activity, rumination, disease occurrence, and management actions. Other (economic and weather) data can be accessed from remote sources using a unified interface that can shield applications from the details of collection and cleaning. The $\mathrm{AgDH}$ is designed as a solution to the problem of managing and integrating all of these different data streams and as a system to ingest and link agricultural data from multiple sources so that relationships between these data sources are established and the data are available from a single source (see Figure 2).

Currently, the data collected from cooperating farms and other sources are stored primarily in raw heterogeneous file formats. As such, the first step is to develop an extraction, transformation, and loading (ETL) process that will prepare the data for access via a programmatic interface. A key component of this process is the

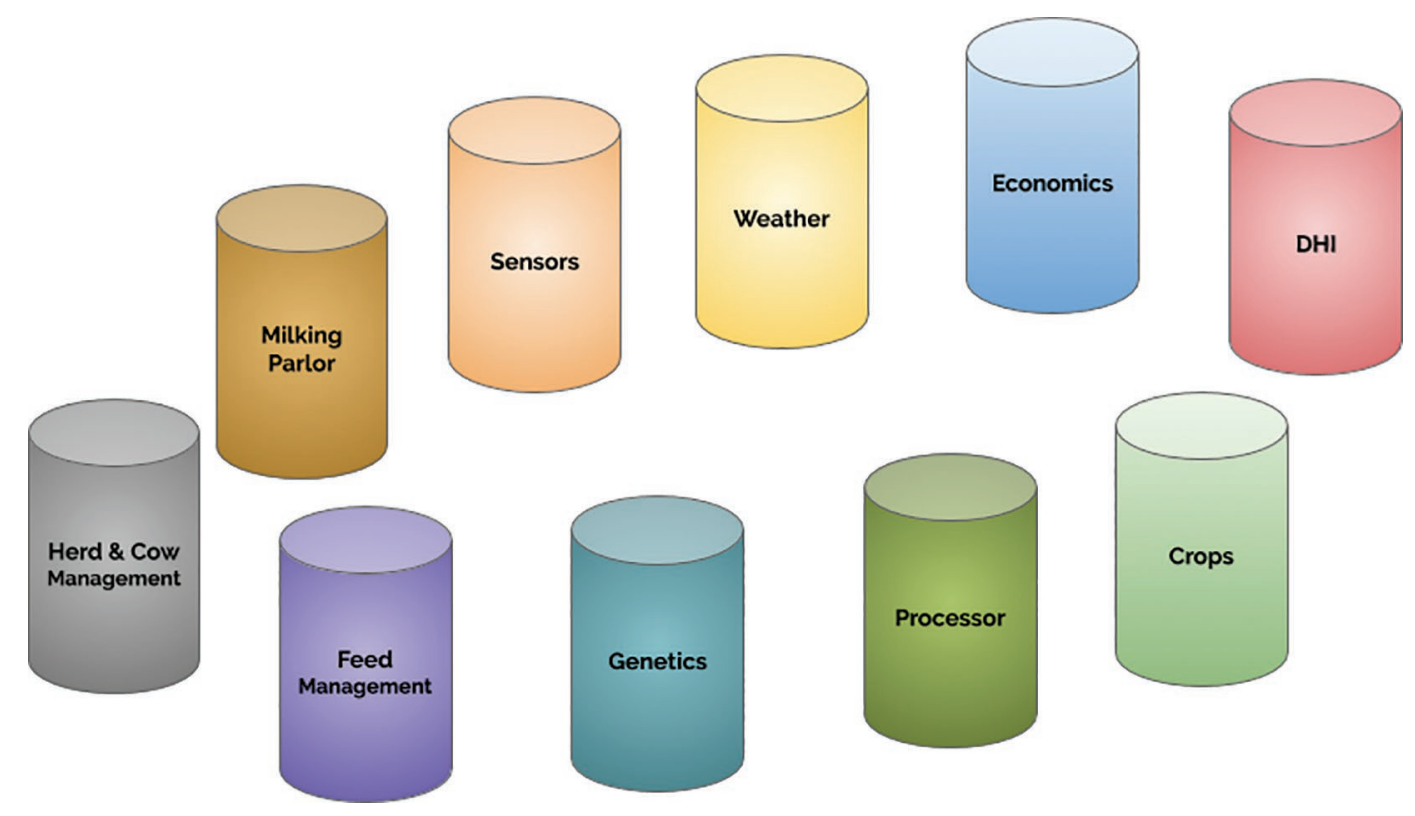

Figure 1. Conceptual model for siloed data sources. 
treatment of missing, corrupt, or inaccurate data. It is clear that missing or dirty data are the norm rather than the exception in farming and many other settings. Missing data can take 2 general forms: unit level (that can be thought of as a failure to take a survey), which can be handled via an appropriate weighting, and the more pervasive situation of item non-response. In this latter case, standard methodologies include listwise deletion (that excludes the whole item for which one data field is missing) or pairwise deletion (that excludes items only if the field is missing in the needed calculation) and mean imputation (replacing a missing field by its mean value). These techniques are ad hoc, and statisticians have shown they are likely to be biased or inefficient. Instead, newer and more principled methods are favored by the statistical community and these include multiple-imputation, full information maximum likelihood, expectation maximization, and matrix completion. An excellent survey is found in Dong and Peng (2013). The power of these methods comes from the fact that they consider conditions under which missing data occurred and combine that information with statistical assumptions. The database community has adopted a different approach to dirty data. This ap- proach can be characterized as data cleaning via probabilistic inference (Abedjan et al., 2016; Rekatsinas et al., 2017), where again statistical models of the data process are encoded, but these are typically combined with automatic repairs of the data.

Next, services are developed to homogenize vendorspecific reporting, making the data consistently available for retrieval and analysis. This involves creating and utilizing a universal relational framework with common keys to query the data (e.g., all relevant data sources about a single cow are linked by a single cow ID). A major task of the AgDH is to perform entity matching so that each individual cow can be identified and matched in different data sources, for example. Entity-matching logic has been developed through consultation with experts. However, as the number of data sources grow, it might be necessary to incorporate a probabilistic or machine learning-assisted approach to merging data. These tools, such as DeepMatcher (https://github.com/anhaidgroup/deepmatcher), could be implemented across the $\mathrm{AgDH}$ (Konda et al., 2016; Mudgal et al., 2018). The hub also needs to create a taxonomy for events that are captured in the data (Schuetz et al., 2018), particularly in animal

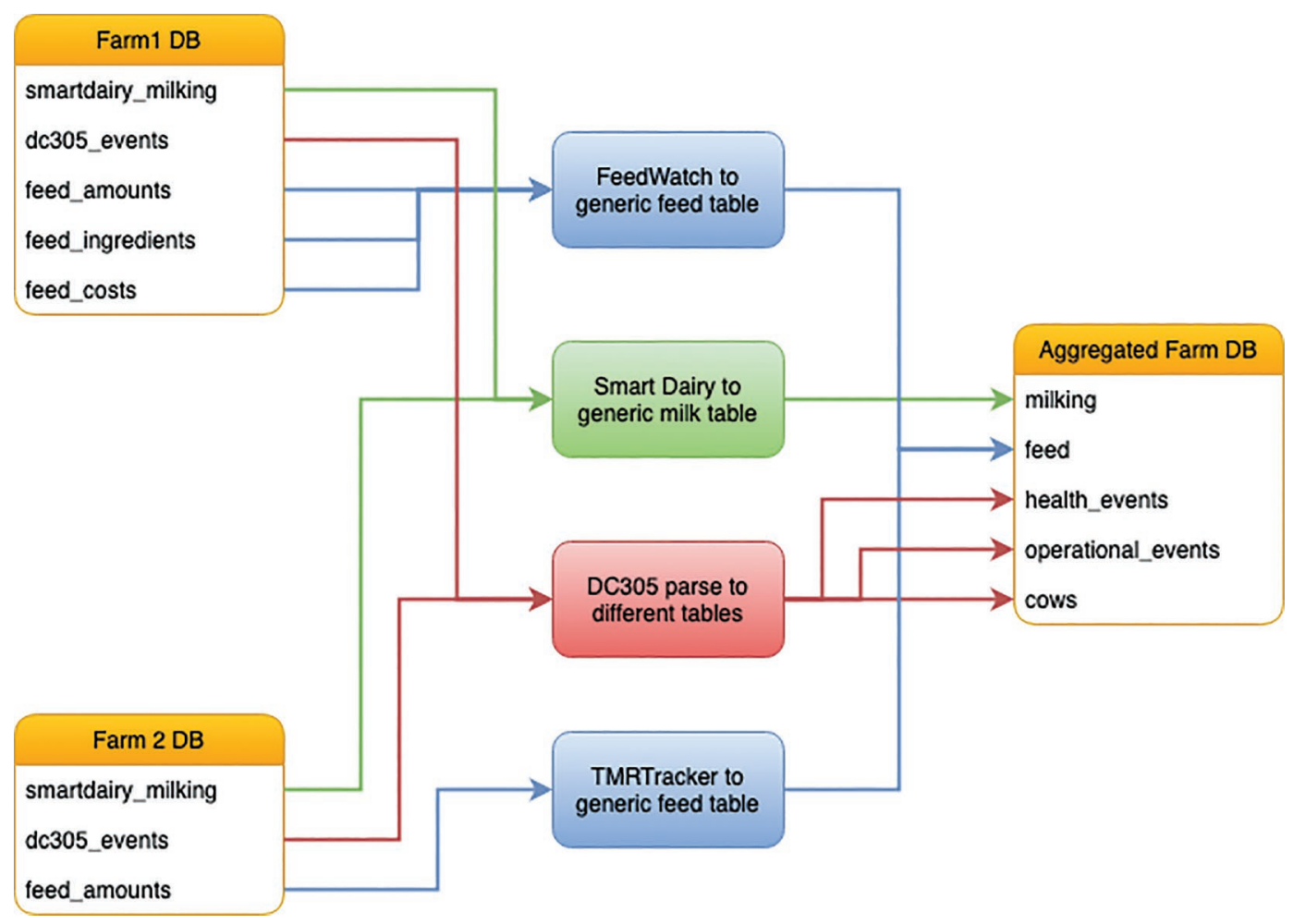

Figure 2. Model data flow from collection points at 2 farms, through a data processing/harmonization system, and deposition into an aggregated database (DB) for use in various forms of decision-support tools. This example highlights the use of heterogeneous assemblages of on-farm software products; for example, both Farm1 and Farm2 in this example use the same parlor software and event tracking software (SmartDairy, https://boumatic.com/eu_en/products/smartdairy, and DC305, https://web.vas.com/dairycomp/, respectively), but have different data representations of feed data (FeedWatch, http://king.vas.com/feedwatch.jsp, vs. TMRTracker, https://digi-star.com/products/details/73/TMR _Tracker), and therefore require different ingest methods. 
health data. The data are then normalized to a consistent format and structure regardless of the source, so that data from one milking parlor software, for example, resembles that from another. A centralized data storage structure is justified in this case because some of these data sources are ephemeral in nature (with data, in some cases, stored at the source for only a few days), along with its heterogeneous and potentially unstructured nature. These data are then loaded into a database that serves as the data warehouse.

\section{APPLICATION PROGRAMMING INTERFACE DESIGN}

Central to the design of our system is the linking of services via application programming interfaces (APIs). These have become a well-accepted approach to providing programmatic access to data and services, and they typically operate by passing a request for data or services through a standard http request along with the required input data. This is the same as requesting a web page in a browser, except instead of an HTML document rendered by a web browser, the response is typically provided in JSON (JavaScript Object Notation), CSV (comma-separated values), or other XML (eXtensible Markup Language) style, which is a humanreadable structured data format. There are 2 primary benefits to the use of APIs: (1) the structured nature clearly defines what the input and outputs are, not only in terms of what information needs to be conveyed, but what explicit data types are expected, and what structure the data is in; and (2) although the request and response structure are rigid in their nature, the ability to provide multiple API endpoints to allow access to different data sources and services overall makes for more flexibility within the system. This permits the development of a modular system, where analytical tools can be developed and deployed independently of each other or the data source. This modularity provides flexibility and freedom for any component to implement their analyses using the most appropriate tools necessary; they can deploy deep learning models developed using TensorFlow and SciKit Learn in a Python environment, deploy MapReduce algorithms or Hadoop implementations in Java, or custom implementations in $\mathrm{C}++$. The specifications provided by the API allow implementation of the analysis to exist independently of the data source.

In our specific approach, both the $\mathrm{AgDH}$ and the Dairy Brain services will be accessible via APIs, as shown in Figure 3. Our primary design is that data from the AgDH (accessible via API calls) will be used to populate analyses provided by the Dairy Brain services. However, the Dairy Brain services are not explicitly tied to the $\mathrm{AgDH}$ to get that data. Should another source be able to provide a similar data source, the Dairy Brain service could easily tie into that third party as an alternative (or potentially even supplemental) data source. Similarly, the AgDH could (and is designed to) provide data access to third-party analytical tools and even researchers. This does not mean that the data will be accessible by just anyone; the nature of the API design allows for finer control, and access to any and all data via this API will be handled via a secure and encrypted https connection. Third parties must be registered and approved by the AgDH service before being provided a token-based authentication process that can be used to individualize and monitor data access.

\section{DAIRY BRAIN}

The development of analytical modules and decision support tools will be collectively referred to as the Dairy Brain. The deliverables of the Dairy Brain center on the development and application of new analytical techniques and the operationalization of these techniques for streaming data. As the number of applications increases and the service is more widely used, computational bottlenecks may be encountered. At that stage, our design allows for parallel implementations of the analytical techniques ("distributed analytics") utilizing the University of Wisconsin-Madison's high-throughput Condor system. The Dairy Brain will also serve as an exemplar of how multiple data sources can be connected through the $\mathrm{AgDH}$ and delivered to advanced analytics to generate value. The analytical pipeline results will become a new source of data that could then be made available directly from the Dairy Brain via access points provided either for programmatic access via APIs or through a web-based user interface. The overall design is depicted schematically in Figure 4.

The Dairy Brain will provide a suite of applications that use all 3 types of analytics: descriptive, predictive, and prescriptive. Descriptive analytics often take the form of key performance indicators (KPIs), which in the dairy industry might include income over feed costs, feed efficiency measures, or disease occurrence. Predictive analytics can also take the form of the same KPIs used in descriptive analytics but involve forecasts of what they are likely to be in the future, rather than a summarization of their values in the past (Morota et al., 2018). Prescriptive analytics often tackle more complicated decisions and tradeoffs and can integrate one or more predictive analyses or data streams to inform how to best reach a goal. Outputs from prescriptive analyses are typically more similar to suggested courses of action or decisions, rather than performance evaluations or predictions. An example could be to suggest 
what herd size and composition would be the most economically efficient. These analyses are often interrelated: the previous example of herd composition and size may be informed by using predictive analyses, such as the predicted productivity of individual cows, in concert with a predictive model of market conditions.

In developing value-added analytical capabilities for the dairy industry, it is imperative that both the $\mathrm{AgDH}$ and the Dairy Brain retain a high level of flexibility, even if it introduces a higher level of complexity (i.e., multiple connections to different data sources). The flexibility retained by developing a network of API connections allows Dairy Brain modules to adapt to industry-wide trends and evolving data landscapes; if advantageous, the Dairy Brain modules could also be redirected to a singular source of data (an $\mathrm{AgDH}$-like service). This development strategy encourages innovative developments in dairy analytics in parallel with the development of fully featured and centralized data handling that maintains elastic data sourcing.

The interchangeable use of test data in the initial application prototype and real data from the $\mathrm{AgDH}$ will facilitate reproducible research. We will be able to verify original research results while moving to a broader collection of farm and data streams.
Maintaining a flexible architecture is a critical design component to maintain relevance in an evolving digital landscape. However, flexibility does not address challenges with scaling an analytic service to a broad base of users. The challenges associated with scaling a service are certainly multifaceted, but when building an API-based system where data integration and processing is the primary value-add, there must be a high level of coordination between participating entities to begin with. In essence, an ontology must exist between data generators along the entire milk product value chain (Tomic et al., 2015). If a formalized ontology does exist, as it does in other sectors such as banking, a high degree of data interoperability can be achieved. This common base language requires participants to come together and recognize that data coordination can be the tide that raises all ships. This integrative effort must, of course, include prime members of the dairy industry, but this effort should not exist within a bubble. It would be valuable to develop this language with entities that interface with the dairy industry - regulatory bodies, financing, logistics, outsourcing, and so on. The idea is that integrative data can be deeply technical but must also facilitate the translation of research into applications that can be understood by an informed lay

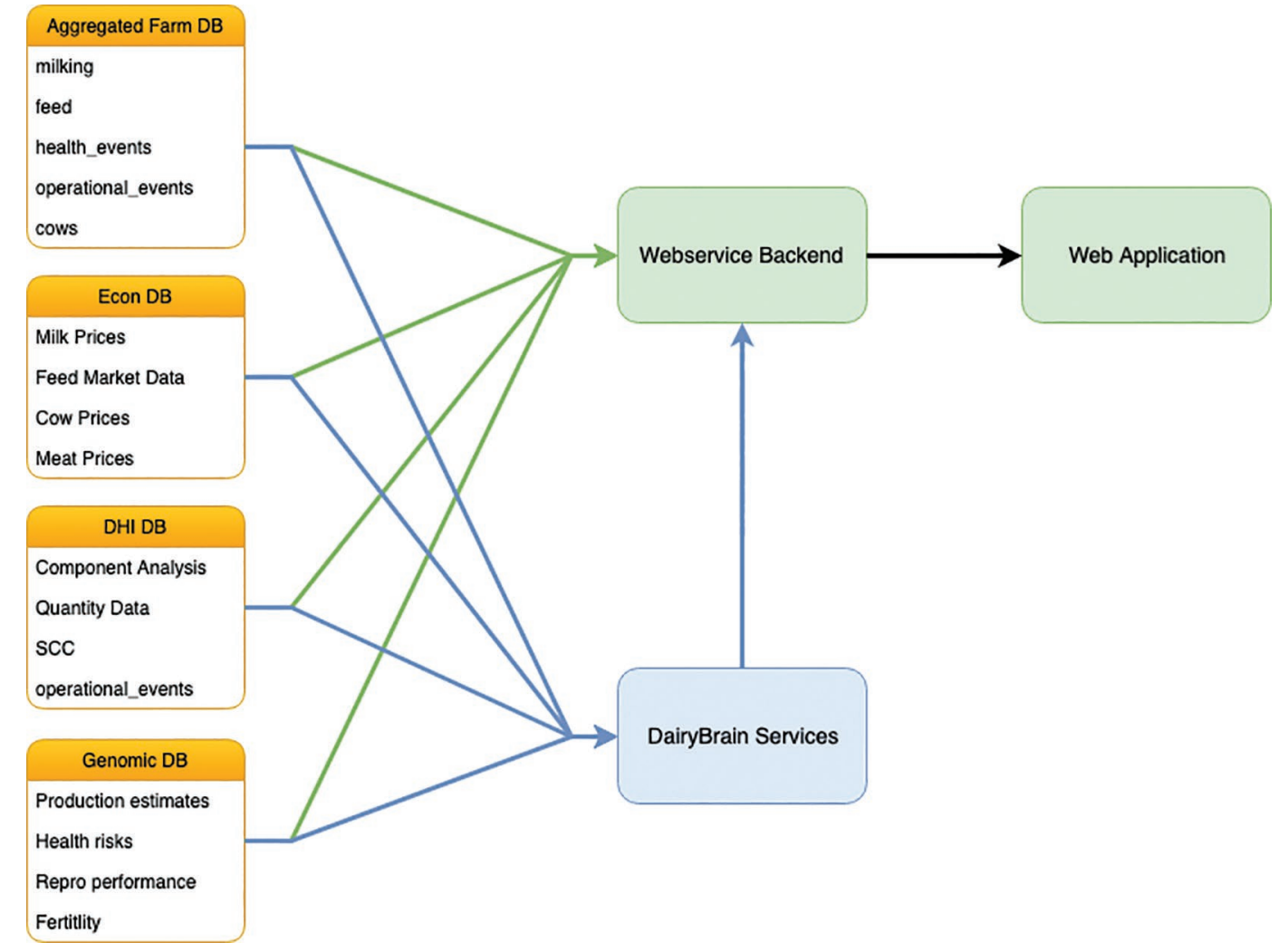

Figure 3. Network of application programming interface (API) connections between the database (DB) backbone and various services (e.g., analytical, web/visualization). 
population - all with the goal of enhancing all aspects of operations (i.e., increase farm value). Developing an ontology is a technical challenge but not an insurmountable one, as evidenced in the successful use by the International Committee for Animal Recording's (ICAR) Interbull initiative for recording breed codes. The industry has achieved a certain momentum that suggests that the dairy sector will continue to embrace more data rather than less. These data will continue to be more specific to individual animals as robotic milkers and custom feeding systems are used in conjunction with remote monitoring systems and health trackers.

The trend toward data-informed dairy operations has arisen largely from farmer-led initiatives. Farmers have complicated business decisions to make daily that exist at multiple scales and a farmer may not have the time or expertise to develop a fully informed response strategy. Operational (short-term) decisions would be better informed by business-relevant KPIs such as daily income over feed costs (milk revenue minus feed cost price, ultimately expressed as $\$ /$ cow per day) or daily feed efficiency (the amount of energy-corrected milk produced by one unit of feed supplied). However, the calculation of these simple descriptive statistics requires highly integrated data streams. Without a data ontology, the informative power of these metrics suffers. Perhaps only averaged values over a pen or the entire herd are available or maybe these values are available on a less-than-ideal periodic basis - it is impossible to make a decision about the relative importance of a cow when only aggregate parameters are available. Pushing integrated data streams beyond the realm of the descriptive and into the world of the predictive and prescriptive will require additional data as well as an even higher level of integration. These data goals might seem to exist only in a distant future. However, research progress in several areas, such as early disease detection (ketosis and mastitis; Ehret et al., 2015; Weigel et al., 2017), probabilistic cow-life value projections (Cabrera, 2010; De Vries, 2006), and strategic pen management (nutritional grouping; Cabrera and Kalantari, 2016; Wu et al., 2019) means that numerous opportunities exist to inject a data-informed decision process into the current dairy market.

\section{SUMMARY}

We have described the flexible design of a Dairy Brain that is coupled via an API to a hub of agricultural data. The flexible design allows many Dairy Brain modules to be developed independently ("horses for courses"), each working with combinations of data streams and models to provide specific recommendations to decision makers. The design facilitates a breadth of applications to be fed by systematically cleaned data from many sources to tailored data analytics capabilities. Research challenges that remain include dealing with increased variability as predictions go from herd or pen level down to individual cow level, and the appropriate choice of tool or technique to deal with a specific problem. Additionally, tools and policies for data cleaning, data curation, and data sharing are urgently needed. We see the need to create a coordinated innovation network (CIN) with representation from multiple academic disciplines and industry. The primary goal of the CIN is to develop a series of design documents outlining the characteristics and structure for operating an ecosystem of services exchanging data within the dairy

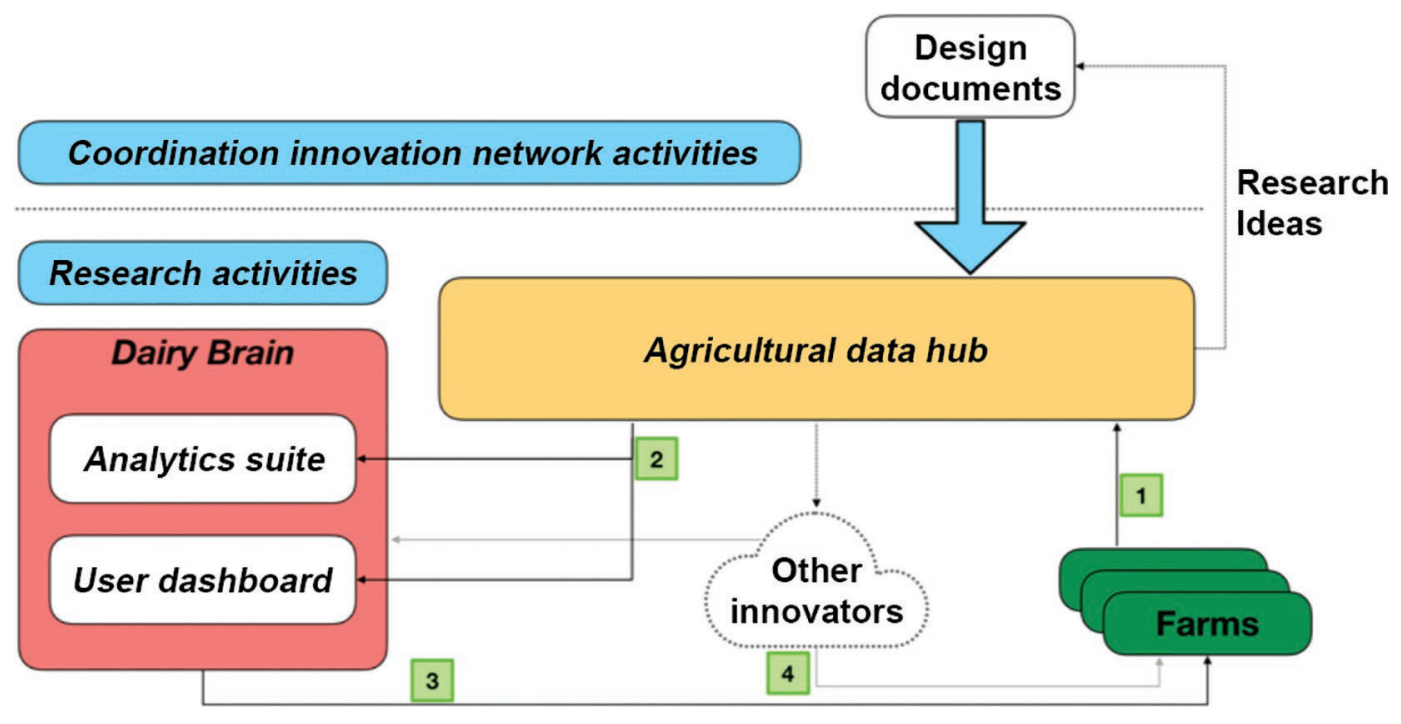

Figure 4. Coordinated innovation network (CIN) design structure. 
industry. The CIN will need to explore issues around data ownership, data security, and the technological and economic aspects of integrating data sources and services from multiple entities in a sustainable way. The deliverables of the CIN are 2-fold: (1) an established network of interested parties with broad representation from the dairy industry, including other potential users of the AgDH ("other innovators" in Figure 4); and (2) a suite of design documents developed by this network that will shape the structure and implementation for the development of the AgDH and Dairy Brain services (Figure 4), and will serve as a basis for broader industry conversations and implementation of services, standards, and designs.

\section{ACKNOWLEDGMENTS}

This project has been supported by the University of Wisconsin-Madison UW2020 initiative that encourages highly innovative and groundbreaking research. This project has also been supported by the USDA-National Institute for Food and Agriculture (Washington, DC; FACT grant 2018-09256). The authors thank Jorge A. Barrientos-Blanco, Victor Cabrera, Hector Delgado, Liliana Fadul-Pacheco, Miron Livny, Jignesh Patel, Kent Weigel, and Heather White (all at the University of Wisconsin-Madison) for their contributions to this work as part of the Dairy Brain team. The authors have not stated any conflicts of interest.

\section{REFERENCES}

Abedjan, Z., X. Chu, D. Deng, R. C. Fernandez, I. F. Ilyas, M. Ouzzani, P. Papotti, M. Stonebraker, and N. Tang. 2016. Detecting data errors: Where are we and what needs to be done? Proc. VLDB Endowment 9:993-1004. https://doi.org/10.14778/2994509 .2994518

Cabrera, V. E. 2010. A large Markovian linear program to optimize replacement policies and dairy herd net income for diets and nitrogen excretion. J. Dairy Sci. 93:394-406. https://doi.org/10.3168/ jds.2009-2352.

Cabrera, V. E., and A. S. Kalantari. 2016. Economics of production efficiency: Nutritional grouping of the lactating cow. J. Dairy Sci. 99:825-841. https://doi.org/10.3168/jds.2015-9846.

De Vries, A. 2006. Economic value of pregnancy in dairy cattle. J. Dairy Sci. 89:3876-3885. https://doi.org/10.3168/jds.S0022 -0302(06)72430-4.

Dong, Y., and C.-Y. J. Peng. 2013. Principled missing data methods for researchers. Springerplus 2:222. https://doi.org/10.1186/2193 $-1801-2-222$

Ehret, A., D. Hochstuhl, N. Krattenmacher, J. Tetens, M. S. Klein, W. Gronwald, and G. Thaller. 2015. Use of genomic and metabolic information as well as milk performance records for prediction of subclinical ketosis risk via artificial neural networks. J. Dairy Sci. 98:322-329. https://doi.org/10.3168/jds.2014-8602.

Konda, P., S. Das, G. C. P. Suganthan, A. Doan, A. Ardalan, J. R. Ballard, H. Li, F. Panahi, H. Zhang, J. Naughton, and S. Prasad. 2016. Magellan: Toward building entity matching management systems. Proc. VLDB Endowment 9:1197-1208. https://doi.org/ $10.14778 / 2994509.2994535$.

Morota, G., R. V. Ventura, F. F. Silva, M. Koyama, and S. C. Fernando. 2018. Machine learning and data mining advance predictive big data analysis in precision animal agriculture. J. Anim. Sci. 96:1540-1550. https://doi.org/10.1093/jas/sky014.

Mudgal, S., H. Li, T. Rekatsinas, A. Doan, Y. Park, G. Krishnan, R. Deep, E. Arcaute, and V. Raghavendra. 2018. Deep learning for entity matching: A design space exploration. Pages 19-34 in Proc. SIGMOD'18: 2018 International Conference on Management of Data, Houston, TX, USA. Association of Computing Machinery, New York, NY. https://doi.org/10.1145/3183713.3196926.

Rekatsinas, T., X. Chu, I. F. Ilyas, and C. Ré. 2017. HoloClean: Holistic data repairs with probabilistic inference. In Proc. Int. Conf Very Large Data Bases (VLDB), Munich, Germany. http://www .vldb.org/pvldb/vol10.html.

Schuetz, C. G., S. Schausberger, and M. Schrefl. 2018. Building an active semantic data warehouse for precision dairy farming. J. Organ. Comput. Electron. Commerce 28:122-141. https://doi.org/10 $.1080 / 10919392.2018 .1444344$.

Tomic, D., D. Drenjanac, S. Hoermann, and W. Auer. 2015. Experiences with creating a precision dairy farming ontology (DFO) and a knowledge graph for the data integration platform in agriOpenLink. J. Agric. Inform. 6:115-126. https://doi.org/10.17700/ jai.2015.6.4.213

Weigel, K. A., R. S. Pralle, H. Adams, K. Cho, C. Do, and H. M. White. 2017. Prediction of whole genome risk for selection and management of hyperketonemia in Holstein dairy cattle. J. Anim. Breed. Genet. 134:275-285. https://doi.org/10.1111/jbg.12259.

Wilkinson, M. D., M. Dumontier, I. J. Aalbersberg, G. Appleton, M. Axton, A. Baak, N. Blomberg, J. W. Boiten, L. B. da Silva Santos, P. E. Bourne, J. Bouwman, A. J. Brookes, T. Clark, M. Crosas, I. Dillo, O. Dumon, S. Edmunds, C. T. Evelo, R. Finkers, A. Gonzalez-Beltran, A. J. G. Gray, P. Groth, C. Goble, J. S. Grethe, J Heringa, P. A. C. 't Hoen, R. Hooft, T. Kuhn, R. Kok, J. Kok, S. J. Lusher, M. E. Martone, A. Mons, A. L. Packer, B. Persson, P. Rocca-Serra, M. Roos, R. van Schaik, S.-A. Sansone, E. Schultes, T. Sengstag, T. Slater, G. Strawn, M. A. Swertz, M. Thompson, J. van der Lei, E. van Mulligen, J. Velterop, A. Waagmeester, P. Wittenburg, K. Wolstencroft, J. Zhao, and B. Mons. 2016. The FAIR Guiding Principles for scientific data management and stewardship. Sci. Data 3:160018. https://doi.org/10.1038/sdata.2016.18.

Wolfert, S., L. Ge, C. Verdouw, and M.-J. Bogaardt. 2017. Big data in smart farming - A review. Agric. Syst. 153:69-80. https://doi.org/ 10.1016/j.agsy.2017.01.023.

Wu, Y., D. Liang, R. D. Shaver, and V. E. Cabrera. 2019. An income over feed cost nutritional grouping strategy. J. Dairy Sci. 102:4682-4693. https://doi.org/10.3168/jds.2018-15302.

\section{ORCIDS}

Michael C. Ferris @ https://orcid.org/0000-0002-7652-6776 